\title{
SmartSantander: Internet of Things Research and Innovation through Citizen Participation*
}

\author{
Verónica Gutiérrez ${ }^{1}$, Jose A. Galache ${ }^{1}$, Luis Sánchez ${ }^{1}$, Luis Muñoz ${ }^{1}$, \\ José M. Hernández-Muñoz ${ }^{2}$, Joao Fernandes ${ }^{3}$, and Mirko Presser ${ }^{3}$ \\ ${ }^{1}$ Universidad de Cantabria, Santander, Spain \\ ${ }^{2}$ Telefonica I+D, Madrid, Spain \\ ${ }^{3}$ Alexandra Institute; Aarhus, Denmark \\ \{veronica, jgalache, lsanchez, luis\}@tlmat.unican.es, jmhm@tid.es, \\ \{joao.fernandes, mirko.presser\} @alexandra.dk
}

\begin{abstract}
The Smart City concept relates to improving efficiency of city services and facilitating a more sustainable development of cities. However, it is important to highlight that, in order to effectively progress towards such smart urban environments, the people living in these cities must be tightly engaged in this endeavour. This paper presents two novel services that have been implemented in order to bring the Smart City closer to the citizen. The Participatory Sensing service we are proposing exploits the advanced features of smartphones to make the user part of the ubiquitous sensing infrastructure over which the Smart City concept is built. The Augmented Reality service is connected to the smart city platform in order to create an advanced visualization tool where the plethora of available information is presented to citizens embedded in their natural surroundings. A brief description of the smart city platform on top of which these services are built is also presented.
\end{abstract}

Keywords: IoT, smart city, augmented reality, participatory sensing.

\section{Introduction}

Smart cities exploit synergies between the ubiquitous sensing technology and their social components to enhance the quality of life of citizens and to improve the efficiency of the city services. In this sense, the Smart City concept [1] and [2] has typically been associated with an eco-system where technology is embedded everywhere. Thus, the different city services (e.g. traffic, water, sewage, energy, commerce, etc.) are greatly improved by exploiting the interconnected information and actuation capabilities that this technology provides.

However, sometimes this technological environment leads us to disregard the fact that the ultimate aim of the Smart City concept must be the citizens. It is important to avoid focusing only on the technology and missing the engagement of society within this paradigm. Smart cities are not simply those that deploy ICT. They need to combine modern technology with smart new ways of thinking about technologies' role in organization, design and planning. As smart city initiatives are planned, the way that

\footnotetext{
* Invited Paper.
}

A. Galis and A. Gavras (Eds.): FIA 2013, LNCS 7858, pp. 173-186, 2013.

(C) The Author(s). This article is published with open access at link.springer.com 
technology can create new urban user experiences must be envisioned. Thinking about the Smart City as a holistic system and considering the manner in which those new systems can bring in positive behavioural change, needs the citizens to become involved from the very first moment of the city smartening process.

This paper presents architecture that, following Internet of Things (IoT) precepts [3] and [4], enables the creation of a ubiquitous sensing infrastructure within the scope of a Smart City aiming at improving city service efficiency. This architecture tackles the challenges pertaining to infrastructure management and data handling. Moreover, it also defines the necessary middleware that enables seamless access to this infrastructure for the development of value-added services. This latter aspect is what mainly motivates this article as two novel services have been developed on top of an urban deployment in the city of Santander, Spain. The deployment consists of a large-scale IoT infrastructure which supports the provision of impact-generating smart city services, directly perceivable by all the Smart City stakeholders [5].

Augmented Reality (AR) systems have recently emerged as a powerful visualization tool, which augments real world elements with digital information. The proliferation of powerful smartphones has accelerated the adoption of AR in mobile environments too. Several AR-based applications have been developed for Android or iOS devices [6] and [7]. Moreover, a particularly important aspect of the AR is its ability to make the user feel naturally surrounded by the technology, thus providing a perfect eco-system for the user to engage with the Smart City concept. In this paper the AR service that has been developed for improving tourist services is described. In this context, the main insights of the service architecture as well as details of the developed AR mobile application are described.

Moreover, mobile phones have evolved from devices that are just used for voice and text communication to platforms that are able to capture and transmit a range of data types (e.g. image, audio, and location). The adoption of these increasingly capable devices has enabled a pervasive sensing paradigm - participatory sensing [8-10]. Participatory sensing systems encourage individuals carrying mobile phones to explore phenomena and events of interest using in situ data collection and reporting. Pertaining to the Smart City scenario, participatory sensing has a twofold advantage. On the one hand, it expands the sensing capabilities that have been deployed in terms of dedicated sensor networks. On the other hand, it makes the citizens feel part of the Smart City in which they all live. These two features have been addressed through the Participatory Sensing service that is described in this paper. Users' mobile phones serve as yet another sensor feeding physical sensing information, e.g. GPS coordinates, noise, temperature, etc. to the platform. Users can also subscribe to services such as "the Pace of the City", where they can get alerts for specific types of events currently occurring in the city. Finally, users can themselves also report the occurrence of such events, which are subsequently propagated to other users who are subscribed to the respective type of events.

The paper is structured as follows. Section 2 describes the architecture and platform on top of which these services have been developed. Particular emphasis is put on the part of the architecture that deals with the service development framework. In Section 3 the AR and Participatory Sensing services are detailed. Moreover, 
the service architecture and the implementation insights are thoroughly described. Finally, conclusions are drawn in Section 4.

\section{Novel Architecture for Service Provision and Experimentation}

The architecture used as reference model for the deployed infrastructure does not solely rely on particular service-specific deployments, but also provides a testbed-like urban experimentation environment for technology and service providers. Although we recognize that the importance of experimentation capabilities in the architecture of a mature smart city of the future may eventually blur, we believe that the early-day Smart Cities will greatly benefit from the capability to experiment towards the development of future services.

The architecture has a three-tiered network approach: IoT node tier, gateway $(\mathrm{GW})$ tier and testbed server tier.

The IoT node tier embraces the majority of the devices deployed in the testbed infrastructure. It is composed of diverse heterogeneous devices, including miscellaneous sensor platforms, tailor-made devices for specific services as well as RadioFrequency Identification (RFID) and Near Field Communications (NFC) tags. These devices are typically resource-constrained and host a range of sensors and in some cases actuators. Other devices such as mobile phones and purpose-built devices with reasonable computing power (e.g. mobile devices in vehicles), as well as providing wide area communication capabilities, behave as IoT nodes in terms of sensing capabilities and as GW nodes regarding processing and communication capabilities.

The GW tier links the IoT devices on the edges of the capillary network to the core network infrastructure. IoT nodes are grouped in clusters that depend on a GW device. This node locally gathers and processes the information retrieved by IoT devices within its cluster. It also manages (transmission/reception of commands) them, thus scaling and easing the management of the whole network. The GW tier devices are typically more powerful than IoT nodes in terms of memory and processing capabilities, also providing faster and more robust communication interfaces. GW devices allow virtualisation of IoT devices. This enables the instantiation of emulated sensors or actuators that behave in all respects similar to the actual devices.

The server tier provides more powerful computing platforms with high availability and directly connected to the core network. The servers are used to host IoT data repositories and application servers. Server tier devices receive data from all GW tier nodes. As a final step, the concept of federation is supported by the architecture. Servers managing networks located in different physical locations can connect among themselves to allow users of the platforms to transparently access IoT nodes that are deployed in different testbeds.

Fig. 1 shows the high-level architecture consolidated in the SmartSantander project [11] and [12] as well as the main functionalities provided and associated with each of the tiers.

The architecture distinguishes four subsystems, namely management, experimentation, application support and, a transverse one, the Authentication, Authorization and 
Accounting (AAA) subsystem. In order to access and interact with these subsystems four interfaces have been defined, named Management support interface (MSI), Experimental Support Interface (ESI), Application Support Interface (ASI) and Access Control Interface (ACI), respectively. For each of the three levels of the architecture, the corresponding functionalities and services associated with the aforementioned four subsystems are implemented.

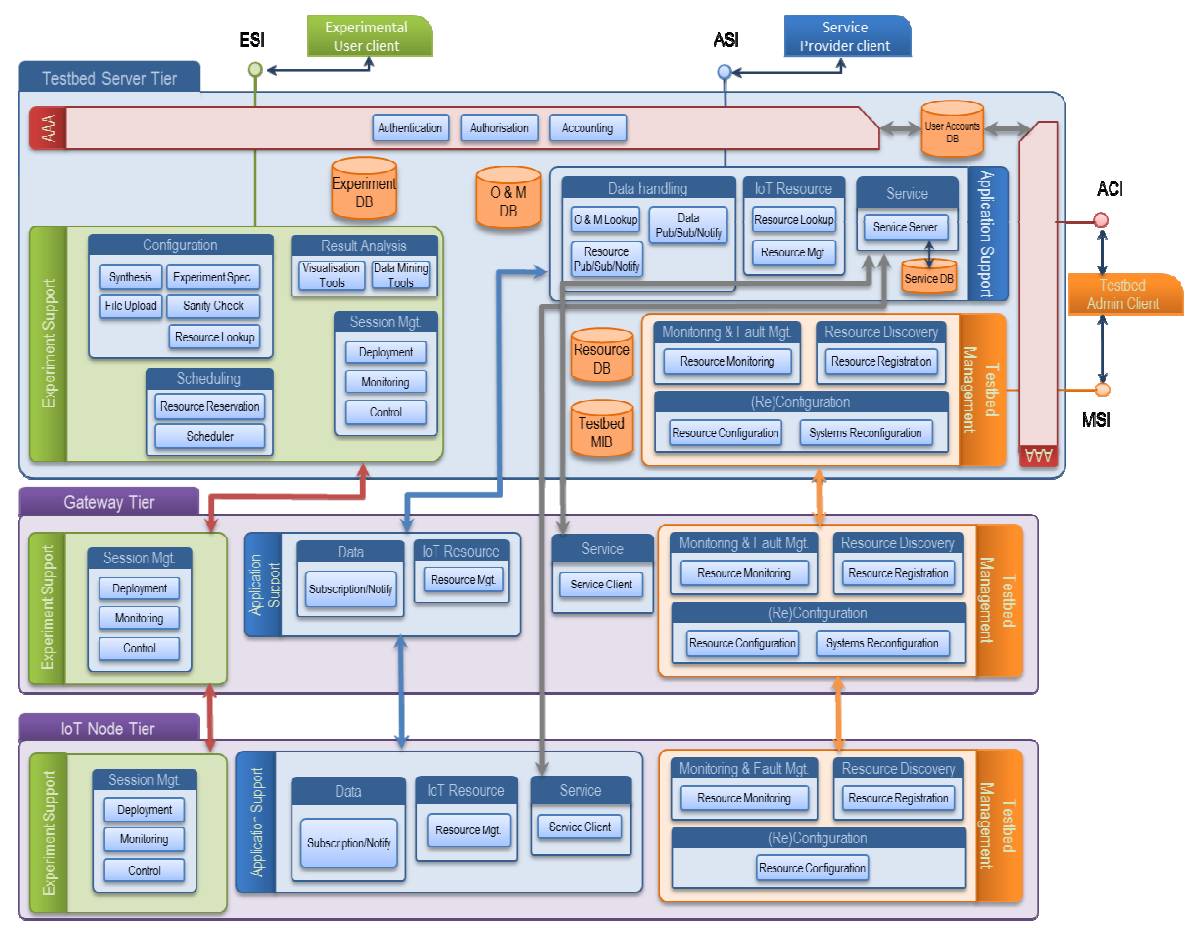

Fig. 1. Platform high-level architecture and building blocks

The Testbed Management subsystem performs three non-trivial management processes, namely: resource discovery, resource monitoring and testbed reconfiguration. The discovery of resources is an essential feature of an IoT platform as it provides support for resource selection according to the user's criteria (for example, sensed phenomena, sensor locality, measurement frequency among others). This essentially entails i) the description of the diversity of IoT resources using a uniform IoT Resource Description Model as well as ii) the generation of these descriptions based on dynamic IoT node registrations, and iii) their lookup based on IoT device attributes, dynamic state and connectivity characteristics. Even under normal operation, the IoT platform is in a constant state of flux: IoT nodes fail, change their point attachment, and join the platform or undergo transition through a number of operation states. Ensuring the correct execution of the IoT testbed's services in the face of such a dynamic environment and guaranteeing the testbed's resilience to failures, requires 
continuous monitoring of the state of its IoT resources. Finally, on the detection of hardware failures, fault-remediation strategies require that the testbed is reconfigured to omit the faulty nodes from future experimentation or service-provisioning.

The Experiment Support subsystem provides the mechanisms required to support different phases of the experiment lifecycle. During the specification phase, which mainly deals with resource selection (i.e. selection of IoT devices and other testbed resources suitable for execution of desired experiment), the user is supported with adequate functionality enabling exploration of available testbed resources (resources are available during the duration of the experiment) aiming at the selection of those fulfilling (in terms of capabilities offered by selected nodes) the desired properties. Once the selected nodes for a determined experiment are reserved and scheduled, they are wirelessly flashed with the corresponding code image. This flashing procedure, carried out through Multihop-Over-The-Air Programming (MOTAP), enables nodes to be flashed as many times and with as many codes as required. Finally, during the execution phase, the experimenter is empowered with the tools for experiment execution control, experiment monitoring, data collection and logging.

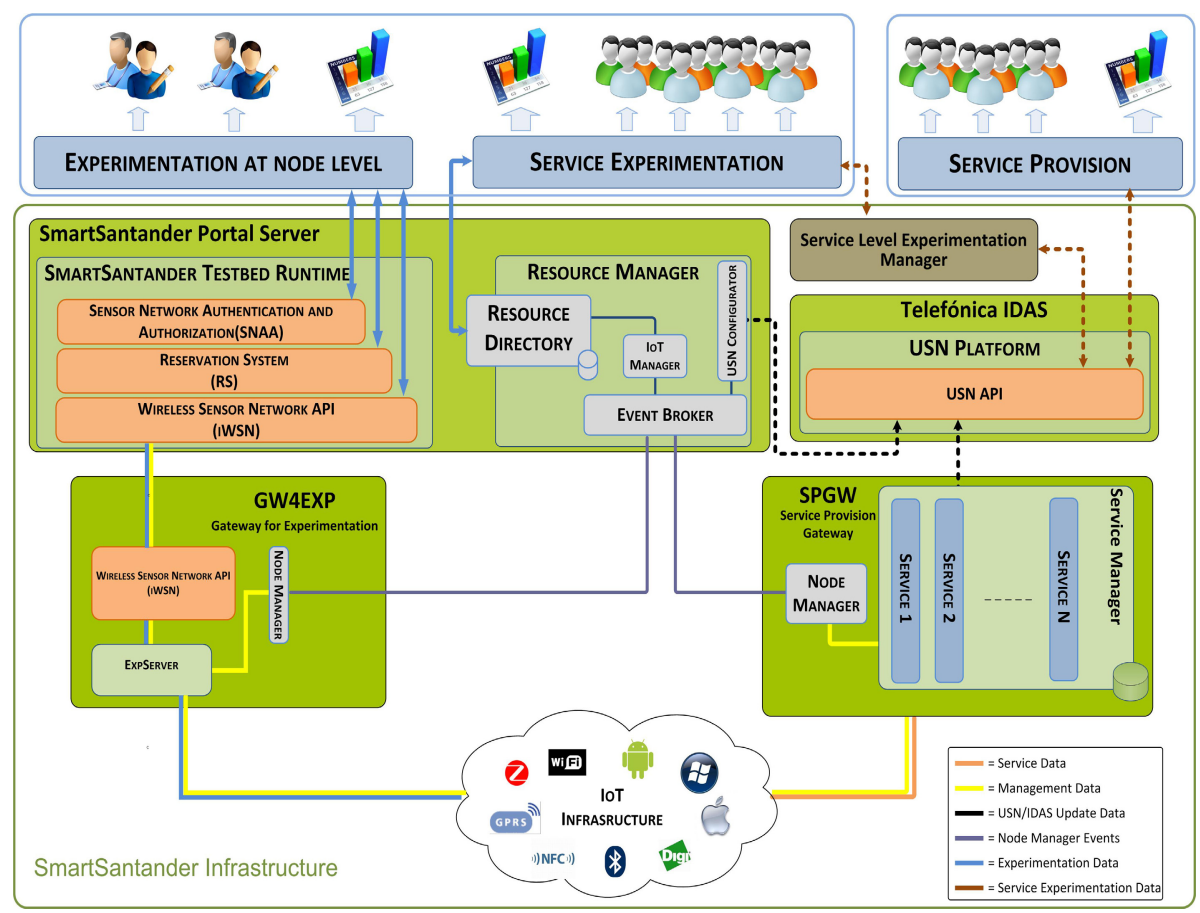

Fig. 2. Low-level platform architecture and implemented modules

Finally, the Application Support subsystem is in charge of providing the functionalities to facilitate the development of services based on the information gathered by the IoT nodes. Besides the storage of the observations and measurements coming 
from the IoT nodes, its main functionalities relate to the lookup and provision of observations to requesting services by means of a publish/subscribe/notify interaction.

At the IoT node and GW level, subscription, notification and resource management are supported, all information retrieved from them being stored in the server tier in the O\&M database. For services taking data retrieved by IoT nodes, the Service client is located in the IoT node tier, whilst for devices acting as GW nodes in terms of communication/processing skills and as IoT nodes providing sensing capabilities, such as mobile phones, the service client is located at GW level. For both cases, information is sent directly from the service client to the service server, storing information in the corresponding database. Finally, information associated with the services is sent from the server tier node to the corresponding Service Provider client through the ASI interface.

Last but not least, the AAA subsystem is in charge of authentication and access control functionalities that are transversally carried out in order to protect all the interaction points that the platform offers to the outside world. This functionality is only carried out at server level in order to grant access to authenticated and authorized experimenters, services providers or testbed administrators.

Fig. 2 shows the low-level architecture that maps the functionalities and services previously described onto specific building blocks. From the user perspective, three main blocks can be identified: service provision, service experimentation and experimentation at node level. Service provision includes the use cases developed within the SmartSantander project, such as Participatory Sensing or AR, among others. These services take information from the IoT infrastructure and process it accordingly to offer the corresponding services. Service experimentation refers to the different experiments/services that can be implemented by external users, utilizing the information provided by the IoT infrastructure deployed within the project. Experimentation at node level implies node reservation, scheduling, management and flashing in order to change behaviour and execute different experiments over a group of nodes, i.e., routing protocol, network coding schemes or data-mining techniques.

In this paper we focus on the description of service provision and service experimentation. To achieve these functionalities, the following main components are identified: Portal Server, Service Provision GW (SPGW), Service-Level Experimentation Manager (SLEM) and Ubiquitous Sensor Network (USN) platform.

The Service Provision GW receives the data retrieved by the deployed devices. All this information is sent and stored in the USN platform. The Node Manager also accesses to this information in order to monitor the available resources and report to the Resource Manager accordingly.

The Portal Server represents the access point to the SmartSantander facility for service providers and experimenters at service level. Through the coordinated action of different building blocks in the GW and server tiers, the Resource Manager keeps the Resource Directory up to date with the available resources within the network

SLEM allows the service-level experimenters (i.e. those running experiments using data provided by deployed nodes) to access data collected from the services. The SLEM allows them to access the USN component providing a number of useful functions for the development of IoT applications and services (e.g. sensor discovery, 
observation storage, publish-subscribe-notify, etc.). For service providers (i.e. those providing a service with data retrieved by the deployed nodes), data generated by nodes within the network is directly accessed through the USN.

\section{Service Provision and Experimentation: Augmented Reality and Participatory Sensing}

The process of evolving towards a Smart City concept implies that besides developing infrastructure new services have to be provided. As final recipients of the developed services and applications, users play a very important role in the conception, design and evaluation phases of such services. This user-driven methodology [13] approach allows, in a first stage, the citizens' problems and needs to be understood, then new and innovative solutions that tackle those needs can be designed and developed and finally those solutions can be evaluated. By following this methodology we increase the impact of these applications and services in the cities. This is the case of the two applications described below, that is, AR and Participatory Sensing.

\subsection{Augmented Reality Service}

In the majority of cities there is a huge amount of information that may be of interest for tourists and citizens but which is not readily accessible because it is so disperse. To avoid that, a new service has been created, unifying the way to access all data sources and presenting them in a context-sensitive, location-aware manner to the end users using AR technology.
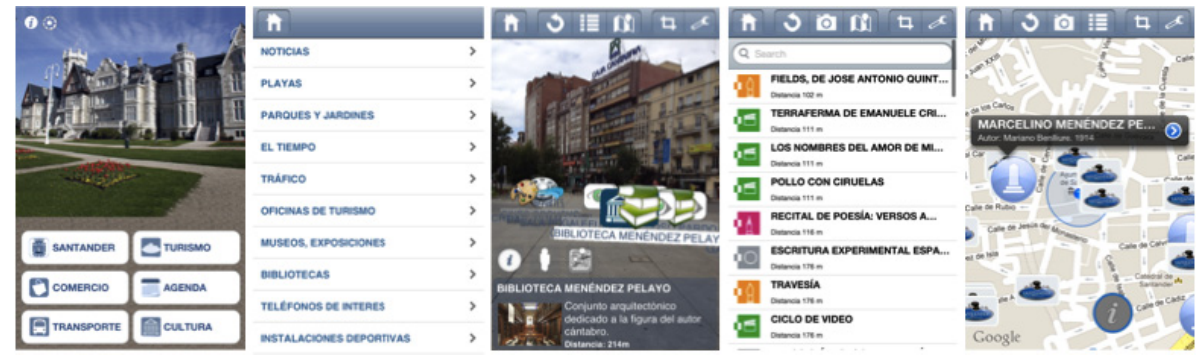

Fig. 3. SmartSantanderRA application screenshots

The AR service developed in the SmartSantander project includes information about more than 2700 places in the city of Santander, classified in different categories: beaches, parks and gardens, monuments, buildings, tourist information offices, shops, art galleries, libraries, bus stops, taxi ranks, bicycle hire points, parking lots and sports centres, as shown in Fig. 3. Furthermore, it allows real-time access to traffic and beach cameras, weather reports and forecasts, public bus information and bike hire service, generating a unique ecosystem for end users when moving around the city. 
As an illustration of the type of service supported by the SmartSantanderRA application [14], it offers an interactive experience through its "stroll in the city" mode. With the application in this mode, visitors will receive information about specific Points Of Interest (POIs) taking into account their preferences as they stroll around the city. This, in general, enhances the serendipity effect for the application end user. In this sense, they can define their own preferences (language, places to visit, etc.) and have an interactive context-sensitive experience visiting the city, rather than using traditional standalone applications.

The deployment of stickers including Quick Response (QR) codes and NFC tags in strategic places in the urban landscape, see Fig. 4, will provide location-sensitive information (transport service, the cultural agenda, shops, monuments, buildings). These stickers link visitors and citizens to the same information included in the AR Service. Additionally, it complements the SmartSantanderRA app, providing precise information about specific POIs.
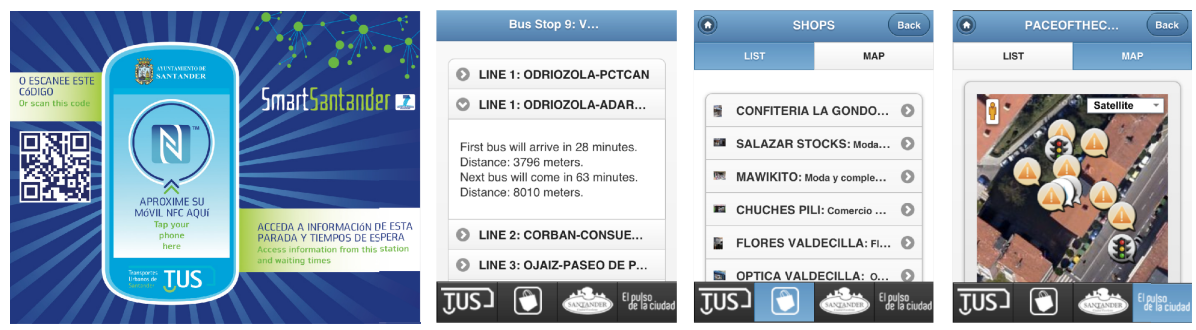

Fig. 4. City guide functionality provided by stickers with NFC technology and QR codes

It is important to note that the AR service has been designed so that the service usage is monitored and the user's behaviour can be analysed, constituting a tool for the creation of new services and experiments within the Smart City context.

\subsection{Participatory Sensing Service}

Nowadays, social media affect a big part of our lives; around $65 \%$ of online adults use social media for personal and professional purposes and $79 \%$ of companies currently use or are planning to use social media [15] and [16]. This increase in use of social media by individuals and companies makes the topic very important for research and development of new and innovative services that can serve both the interests of companies and individuals and can create strong relationships among them.

For this reason we have created the Participatory Sensing service, see application appearance in Fig. 5, which aims at exploiting the use of citizens' smartphones to make people become active in observations and data contribution. In this scenario citizens, Santander City Council and the local newspaper "El Diario Montañés" are connected into a common platform where they can report, share and be notified of events happening in the city. As an example, a user walking in the city centre who finds a hole in the pavement can take a picture, write a text and finally share this incidence with other users of the application. The Santander City Council will therefore 
be notified of the occurrence of the event and proceed accordingly by sending an employee to the location in order to fix this problem. Another example would be when a user reports a road accident; all the other users that are subscribed to this type of event will be notified and can try to avoid driving in this area. By being connected to the Participatory Sensing service, the local newspaper "El Diario Montañés" also enriches this body of knowledge by sharing the daily news information with all the other users of the service. Moreover, the newspaper has created an online information channel called "ElPulsodelaCiudad (Pace of the City)" [17], which provides the citizens with an interface for accessing Participatory Sensing events as well as public transport information, the cultural agenda and sensor values from the SmartSantander IoT infrastructure all on the same website.
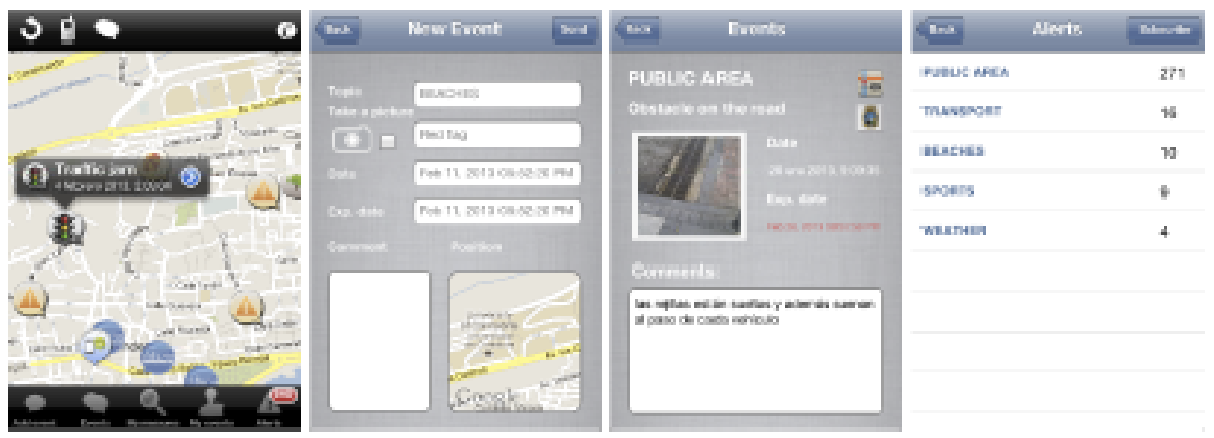

Fig. 5. Pace of the City application screenshots

As well as sharing these social-related events, this application periodically samples the values sensed by the smartphones, such as GPS location, acceleration, temperature, humidity, etc. This information is fed into our platform periodically and can be very valuable in the development of new and innovative services.

\subsection{Service Component Implementation}

Fig. 6 depicts the low-level service provision architecture, showing the interaction between the different components and elements involved in the AR and the Participatory Sensing services. As can be seen, several additional software components are required. SmartSantanderRA mobile applications, AR Server and AR Content Server (ARC Server) can be identified in the AR Service. Besides these, it includes end users with their mobile phones and tags installed in strategic places. Furthermore, two different server components have been developed for the Participatory Sensing Service: the Participatory Sensing Server (PSens Server) and the Pace of the City Server.

Moreover, the USN stores all the data in a common repository, providing an API based on a REST web service for data access. This allows service providers or experimenters to carry out their own developments. In this sense, AR and Participatory Sensing services information can be combined with data collected from the IoT infrastructure in order to create novel and innovative services or experiments. 


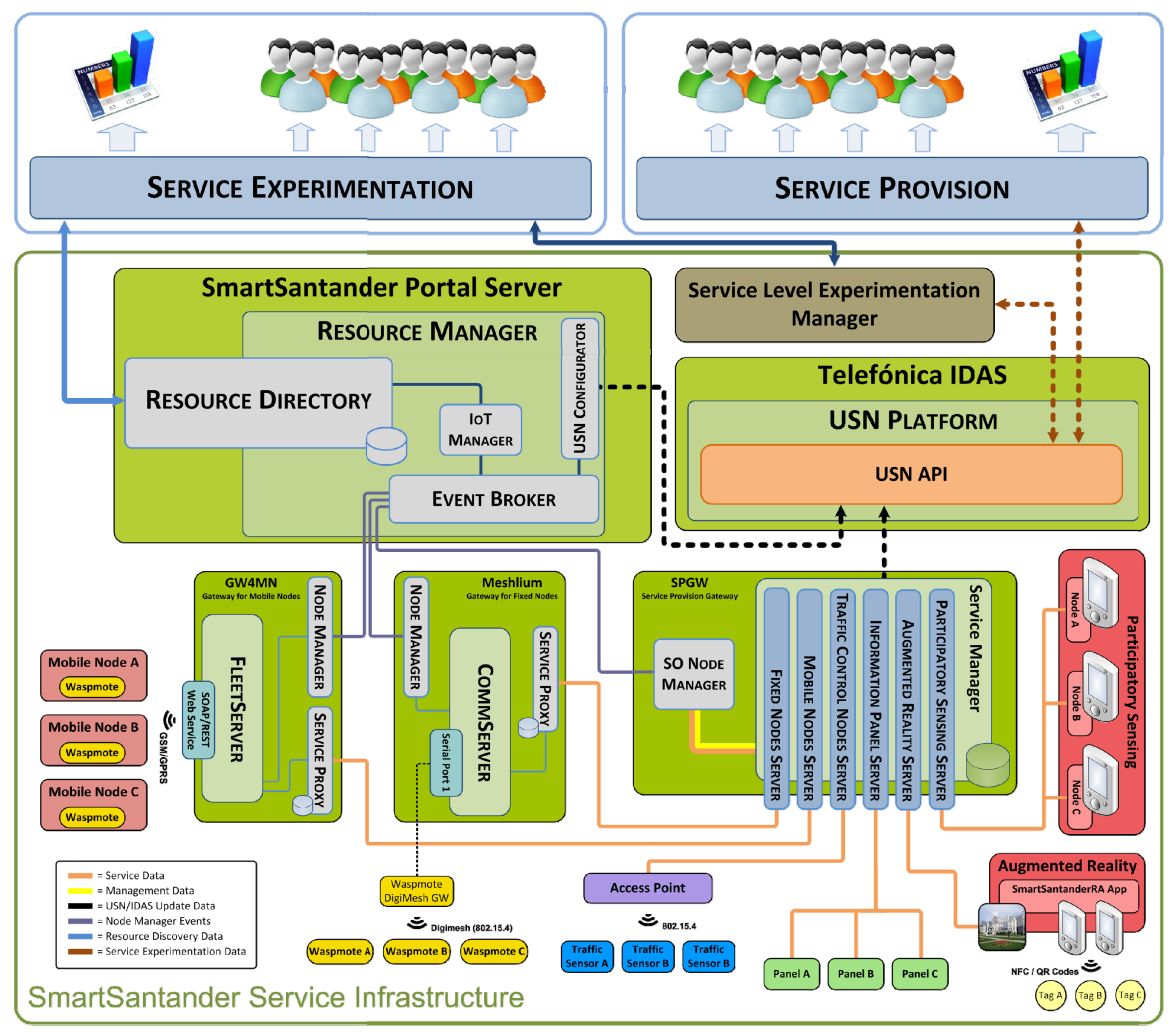

Fig. 6. Low-level service provision architecture

\section{Service Provision Gateway Servers}

The PSens Server and AR Server are the bridge between services and the SmartSantander platform. These components are responsible for registering the mobile nodes in the SmartSantander framework, both PSENS_NODES and AR_NODES, submission of observation messages coming from the applications related to physical sensing, social-related events and retrieval of historical physical sensing information and information generated based on the user behaviour in the AR scenario.

When starting the application for the first time, the mobile device is registered in the SmartSantander platform through the PSens Server or the AR Server. In the case of successful device registration, they return the generated Universal Unique IDentifier (UUID) to the corresponding application. This UUID is stored locally in the mobile phone and will be sent as a parameter in future calls allowing the identification of the device.

\section{Augmented Reality Content Server}

The ARC Server is responsible for returning the requested contents to the SmartSantanderRA application as well as for dynamically generating the mobile web pages. The sequence of actions involving the ARC Server is described below: 
i. Every time the ARC Server receives a request, it communicates to the AR Server, within the Service Provision Gateway, generating messages that will include context information about user behaviour that will enable observations to be sent to the USN.

ii. Once the ARC Server receives a request from the application, it looks for the nearby POIs in its own database, returning the requested information in a unified way, using the ARML v1.0 data format, which is specifically targeted for mobile AR applications.

iii. By reading a NFC tag or QR code, the smartphone sends a request to this server. It will contact legacy systems as well as its own database and return a mobile web page using JQuery Mobile that enhances the mobile web application by integrating HTML5, CSS, JQuery and JQuery UI.

\section{Pace of the City Server}

The Pace of the City Server is responsible for handling subscriptions and socialrelated events. It has its own database with subscriptions and events as well as a PUSH notification component, enabling users to subscribe and unsubscribe to specific types of events. Moreover, it provides a REST service interface with methods to ask about events of a specific type, date, or events nearby the user's current location.

For queries about specific types of events, dates, or even location the mobile applications send the requests to the Pace of the City Server, which retrieves the event information from its local database, matches the events according to the filter and returns the list of filtered events to the application.

\section{SmartSantanderRA Application}

SmartSantanderRA is an application for citizens and visitors available on Android and iOS platforms. It combines AR technology with information existing in the City Council legacy systems, creating an interactive experience when walking along the city, rather than using traditional standalone applications or websites.

Once started the application, it presents a main screen with six options (Santander, Tourism, Commerce, Agenda, Transport and Culture), which provide access to information about different areas of interest in the city.

If the user presses on the AR buttons (Tourism, Commerce, Agenda, Transport, Culture), the AR view will be started, creating on the smartphone screen an overlay on the camera feed with nearby POIs filtered by the selected option. If a particular POI is chosen, further information (title, short description, photo and distance to the POI) is displayed. Moreover, the application allows the user to create the route to that place or to show the street view or playing digital content related to the POI (e.g. videos) if it is available. Besides the AR view, the user has the possibility to access such information by placing POIs on the map view or showing and filtering them within the list view.

More than 10.000 users have downloaded the SmartSantanderRA application since $3^{\text {rd }}$ August 2012. During this time, users are actively using the application, on average generating more than 2000 requests per day to the ARC Server. If accessed information is analysed by POI's category, transport reaches 52 per cent of requests, followed by commerce with 28 per cent and tourism with 17 per cent. 


\section{Pace of the City Application}

The Pace of the City application [18] is available on both Android and iOS platforms. It provides two main functionalities: in the first one the application samples and periodically sends to the PSens Server all the sensor capabilities the smartphone has, which includes: acceleration, temperature, luminosity, noise, battery charge, gravity, pressure, proximity, etc. The second functionality allows the user to create and share the social-related events, subscribe and unsubscribe to specific types of events and to ask about specific events.

Once the application starts, a map view is loaded, in which the user can see his/her current position and, if there are nearby events, they will appear with pins on their location. On the bottom of the screen, five buttons allow the user to: "Add Event" by clicking on this button the user will be able to report an event, the "Events" button allows the user to search for specific events, the filtering can be by event type, date or user location, "My Measures" button allows the user to visualise his historical physical sensing data, "My events" button allows the user to visualise his/her published events and the "Alerts" button allows the user to see his/her current alerts. All the information can be seen either on a map or in a list.

Since November $11^{\text {th }}$, the application has attracted 4,100 downloads. During this time, the citizens have reported more than 1,200 events. 60 per cent of them have notified the municipal services in order to deal with the corresponding incidence. It is important to note that the use of IOT technology has changed the way the City Council deals with the incidences communicated by the citizens. This has caused a complete reorganization of the municipal services. In this sense, the City Council has improved its response to the citizens, reducing the time to find out a solution for an incidence from 38.5 to 14.2 days in the last two months of 2012. During 2013, this indicator has become even better, taking just 5.71 days on average to solve an incidence within Santander.

\section{Conclusions}

The smart city scenario provides the different ecosystem stakeholders (municipality authorities, researchers, etc.) with a unique framework for the creation of sustainable cities by means of new services relying on state-of-the-art technologies. However, the risk of an extensive use of technology magnifying the digital gap for those citizens who do not have the required skills cannot be neglected. Aiming at overcoming this risk, when striving for intelligence at the city level to develop the smart city concept, the citizen-centric approach has to be preserved. Furthermore, we believe that the city scenario offers a fantastic possibility to the research community, companies and individuals to explore and research on new technologies, protocols and services. Bearing in mind all these considerations, the project SmartSantander has built a dual-purpose facility, supporting both experimentation and service provision. The infrastructure is suitable to conduct research activity on top of a massive IoT experimental facility with more than 25,000 fixed and mobile devices. Additionally, two citizen-centric services, named AR and Participatory Sensing, have been developed with the aim of 
intensively involving users in the smart city scenario. The former aggregates the digital information layer made available by the city council and pops it up on citizens' smart phone screens. Additionally, the information implicitly generated by the user is processed by the system with the aim of acquiring knowledge about citizen preferences, mobility patterns and other statistics. The latter makes the concept of the citizen as a sensor a reality. The use of the Participatory Sensing application enables users to report events and incidences and to share them with other users subscribed to the service. Furthermore, there is an option allowing users to enable remote access to some of the physical sensors of his/her mobile terminal, hence helping to provide complementary real-time information about environmental and traffic conditions in different areas of the city.

Of course, the user's privacy always has to be strictly respected in such an interaction, and complete transparency must be provided so citizens are always aware and conscious of the type of information they are contributing with to help create a system that is useful for the community. It is essential for the user to know how to access the option enabled/disabled data sharing, and to be confident that data will be processed in a disaggregated manner, preventing personal information from being bound to retrieved data. Some preliminary surveys show that there is an increasing interest in this type of participatory services, and that a number of people are willing to support them if non-commercial interests can be guaranteed. The first trials have shown that, even with a small proportion of the population taking part in them, data provided through user participation can be extremely useful to complement data gathered from the network of static sensors already deployed throughout the city. This also demonstrates the need for new ways to process and integrate heterogeneous data sources.

Acknowledgements. Although only a few names appear in this paper's list of authors, this work would not have been possible without the contribution and encouragement of the enthusiastic team of the SmartSantander project which has been partially funded by the European Commission under the contract number FP7-ICT-257992.

Open Access. This article is distributed under the terms of the Creative Commons Attribution Noncommercial License which permits any noncommercial use, distribution, and reproduction in any medium, provided the original author(s) and source are credited.

\section{References}

1. Schaffers, H., Komninos, N., Pallot, M., Trousse, B., Nilsson, M., Oliveira, A.: Smart Cities and the Future Internet: Towards Cooperation Frameworks for Open Innovation. In: Domingue, J., et al. (eds.) Future Internet Assembly. LNCS, vol. 6656, pp. 431-446. Springer, Heidelberg (2011)

2. Nam, T., Pardo, T.A.: Conceptualizing smart city with dimensions of technology, people, and institutions. In: Proceedings of the 12th Annual International Digital Government Research Conference, College Park, Maryland, USA, June 12-15, pp. 282-291 (2011)

3. Atzori, L., Iera, A., Morabito, G.: The Internet of Things: A survey. Computer Networks 54(15), 2787-2805 (2010) 
4. Vermesan, O., Friess, P., Guillemin, P., Gusmeroli, S., Sundmaeker, H., Bassi, A., SolerJubert, I., Mazura, M., Harrison, M., Eisenhauer, M., Doody, P.: Internet of Things Strategic Research Roadmap. In: Vermesan, O., Friess, P. (eds.) Internet of Things - Global Technological and Societal Trends. River Publishers, Aalborg (2011)

5. Galache, J.A., Santana, J.R., Gutiérrez, V., Sánchez, L., Sotres, P., Muñoz, L.: Towards Experimentation-Service duality within a Smart City scenario. In: Proceedings of the 9th International Conference on Wireless On-demand Network Systems and Services, Courmayeur, Italy, January 9-11 (2012)

6. Wikitude Augmented reality browser, http://www.wikitude.com/ (accessed on February 10, 2013)

7. AroundMe, http://www. aroundme.com/ (accessed on February 10, 2013)

8. Campbell, A., Eisenman, S., Lane, N., Miluzzo, E., Peterson, R.: People-centric urban sensing. In: Proceedings of the 2nd International Conference on Wireless Communications, Networking and Mobile Computing, Boston, Massachusetts, USA, September 22-24, pp. 18-32 (2006)

9. Burke, J., Estrin, D., Hansen, M., Parker, A., Ramanathan, N., Reddy, S., Srivastava, M.: Participatory sensing. In: Proceedings of 1st Workshop on World-Sensor-Web: Mobile Device Centric Sensory Networks and Applications, Boulder, Colorado, USA, October 31, pp. 1-5 (2006)

10. Paulos, E., Honicky, R., Hooker, B.: Citizen Science: Enabling Participatory Urbanism. In: Foth, M. (ed.) Urban Informatics: The Practice and Promise of the Real-time City, pp. 414-434. IGI Global (2008)

11. SmartSantander project deliverable D1.1, First Cycle Architecture Specification, http: / /www. smartsantander. eu / downloads / Deliverables / D1.1.pdf (accessed on February 10, 2013)

12. SmartSantander project deliverable D1.2, Second Cycle Architecture Specification, http: / /www. smartsantander. eu / downloads / Deliverables / d1.2.pdf (accessed on February 10, 2013)

13. Greenbaum, J.M., Kyng, M.: Design at Work: Cooperative Design of Computer Systems. Routledge (1991)

14. SmartSantanderRA application, http: / /www. smartsantander.eu/index.php/ blog/item/174-smartsantanderra-santander-augmented-realityapplication (accessed on February 10, 2013)

15. PewInternet survey, http: / pewinternet.org/Reports /2011/Social-NetworkingSites.aspx

16. Harvard Business Review, http: //hbr.org/web/slideshows/social-media-what-mostcompanies-dont-know/1-slide (accessed on February 10, 2013)

17. Pace of the city initiative at El Diario Montañés, http: / /www. elpulsodelaciudad. com (accessed on February 10, 2013)

18. Pace of the City application, http://www.smartsantander.eu/index.php/ blog/item/181-participatory-sensing-application (accessed on February 10, 2013) 